\title{
Incorporating directional signs into indoor navigation systems
}

\author{
Wangshu Wang ${ }^{\mathrm{a},}{ }^{*}$, Haosheng Huang ${ }^{\mathrm{b}}$, Hao Lyu $^{\mathrm{c}}$, Georg Gartner ${ }^{\mathrm{a}}$ \\ ${ }^{a}$ Research Group Cartography, Department of Geodesy and Geoinformation, Vienna University of Technology, Vienna, Austria, \\ wangshu.wang@tuwien.ac.at, georg.gartner@tuwien.ac.at \\ ${ }^{b}$ GIScience Center, Department of Geography, University of Zurich, Zurich, Switzerland, haosheng.huang@geo.uzh.ch \\ ${ }^{c}$ Chair of Cartography, Technical University Munich, Munich, Germany, hao.lyu@tum.de \\ * Corresponding author
}

Keywords: Indoor Navigation, Signage, Spatial Knowledge Acquisition, Location Based Services

\begin{abstract}
:
Recent years have witnessed the rapid development of navigation systems. They gradually become the default way of navigating in our daily life. Despite benefiting from their convenience, we may be negatively influenced by them (Parush et al. 2007). Current navigation systems usually draw too much of users' attention to the mobile devices, which leads to users' lacking interaction with the environment. It is often the case that people easily forget travelled route and get lost without navigation systems (Reilly et al. 2008). Empirical study shows that by dividing attention, navigation systems impair users' spatial memory, and thus impact their navigation skills (Gardony et al. 2013). Since a higher engagement with the environment can improve users' spatial knowledge acquisition without decreasing efficiency (Brügger et al. 2017), navigation systems should incorporate important environmental features.
\end{abstract}

As objects of visual, cognitive or structural importance (Sorrows \& Hirtle 1999), landmarks have been extensively studied, and considered as an important environmental feature to be incorporated into navigation systems (Nuhn \& Timpf 2018, Richter 2017, Duckham et al. 2010, Raubal \& Winter 2002). They serve as references to provide users with more natural and pleasant navigating experience (Richter \& Winter 2014). However, most of landmark-related studies are in outdoor environment. Although researchers have attempted to classify and assess indoor landmarks (Ohm 2014, Viaene 2014), it is still very difficult to determine suitable ones. As a subset of semantic indoor landmarks, existing navigational aids (e.g. signs and floor plans) are designed to assist navigation. Among them, signs are most preferred by users (Wang et al. 2017). Being the signs that give necessary information to route users to possible destinations, directional signs contribute most to wayfinding (Gibson 2009). With route information explicitly presented by them, they are relevant environmental features to be incorporated into indoor navigation systems.

In this study, we therefore propose to incorporate directional signs into indoor navigation systems, in order to guide users' attention back to the environment, to facilitate their spatial learning and improve their spatial knowledge acquisition.

We incorporated directional signs into a proof-of-concept indoor navigation system. First, we integrated directional signs and their semantics into a graph-based model. A directional sign was modelled as a node. Each destination on the sign was linked with the sign by an edge. In order to differentiate it from the normal edge, it was marked as semantic edge. Thus, the semantics of the sign was modelled directly into the graph, instead of being stored as attributes in a previous version of our study (Wang \& Gartner 2018). Then, a top-K shortest path algorithm was employed to find candidate routes (Yen 1971). In the end, the route with the largest coverage of semantic edges was suggested to the user. The users would then be explicitly guided to follow the directional signs in the environment.

In the next step, we will evaluate the efficiency and effectiveness of the proposed navigation system by an empirical study with human subjects. Within the experiment, we will also investigate on whether this kind of guidance can succeed in drawing users' attention back to the environment and support their spatial knowledge acquisition.

\section{References:}

Brügger, A., Richter, K. F., \& Fabrikant, S. I. (2017). Distributing Attention Between Environment and Navigation System to Increase Spatial Knowledge Acquisition During Assisted Wayfinding. In International Conference on Spatial Information Theory (pp. 19-22). Springer, Cham

Duckham, M., Winter, S., \& Robinson, M. (2010). Including landmarks in routing instructions. Journal of Location Based Services, 4(1), 28-52. doi:10.1080/17489721003785602

Gardony, A. L., Brunyé, T. T., Mahoney, C. R., \& Taylor, H. A. (2013). How navigational aids impair spatial memory: Evidence for divided attention. Spatial Cognition \& Computation, 13(4), 319-350.

doi: 10.1080/13875868.2013.792821 
Gibson, D. (2009). The wayfinding handbook: Information design for public places. Princeton Architectural Press.

Nuhn, E., \& Timpf, S. (2018). An Overall Framework for Personalised Landmark Selection. In LBS 2018: 14th International Conference on Location Based Services (pp. 231-253). Springer, Cham.

Ohm, C., Müller, M., Ludwig, B., \& Bienk, S. (2014). Where is the landmark? Eye tracking studies in large-scale indoor environments. In P. Kiefer, I. Giannopoulos, M. Raubal, \& A. Krüger (Eds.), Proceedings of the 2nd International Workshop on Eye Tracking for Spatial Research (Vol. i, pp. 47-51). Vienna, Austria.

Parush, A., Ahuvia, S., \& Erev, I. (2007). Degradation in spatial knowledge acquisition when using automatic navigation systems. In International Conference on Spatial Information Theory (pp. 238-254). Springer, Berlin, Heidelberg.

Raubal, M., Winter, S. (2002) Enriching wayfinding instructions with local landmarks. In: Egenhofer M, Mark D (eds) Geographic Information Science. Second International Conference, GIScience 2002 (pp. 243-259). Berlin, Germany: Springer-Verlag.

Reilly, D., Mackay, B., \& Inkpen, K. (2008). How mobile maps cooperate with existing navigational infrastructure. In Map-based mobile services (pp. 267-292). Springer, Berlin, Heidelberg.

Richter, K. F. (2017). Identifying landmark candidates beyond toy examples. KI-Künstliche Intelligenz, 31(2), $135-139$. doi: 10.1007/s13218-016-0477-1

Richter, K.F., \& Winter, S. (2014). Landmarks. Springer Cham Heidelberg New York Dordrecht London. doi:10.1007/978-3-319-05732-3

Sorrows, M., \& Hirtle, S. (1999). The nature of land-marks for real and electronic spaces. In C. Freska \& D. M. Mark (Eds.), Spatial information theory. Cognitive and Computional Foundations of Geographic Information Science (Vol. 1661, pp. 37-50). Berlin, Germany: Springer-Verlag.

Viaene, P., Vanclooster, A., Ooms, K., \& De Maeyer, P. (2014, November). Thinking aloud in search of landmark characteristics in an indoor environment. In Ubiquitous Positioning Indoor Navigation and Location Based Service (UPINLBS), 2014 (pp. 103-110). IEEE.

Wang, W., Huang, H., \& Gartner, G. (2017). Considering Existing Indoor Navigational Aids in Navigation Services. In International Conference on Spatial Information Theory (pp. 179-189). Springer, Cham.

Wang, W., \& Gartner, G. (2018). Integrating Existing Indoor Navigational Aids into Data Modelling and Route Planning. In Adjunct Proceedings of the 14th International Conference on Location Based Services (pp. $147-$ 151). ETH Zurich.

Yen, J.Y., 1971. Finding the $\mathrm{k}$ shortest loopless paths in a network. Management Science, 17 (11), $712-716$. doi: $10.1287 / \mathrm{mnsc} .17 .11 .712$ 